\title{
Aspirin and new approaches for chemoprevention: can we target cancer stem cells?
}

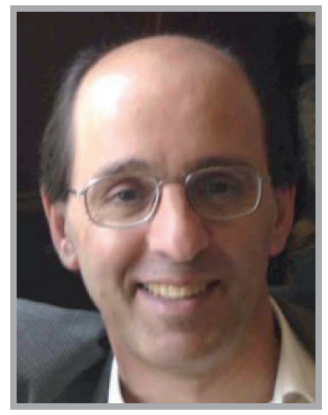

Chris Paraskeva* speaks to Laura Dormer, Commissioning Editor: Chris Paraskeva is Professor of Experimental Oncology and Director of the Cancer Research UK Colorectal Tumour Biology Research Group at the University of Bristol (UK). His academic career has focused on research and educational activities in cellular and molecular biology of colorectal cancer. Professor Paraskeva has had a long standing interest in promoting the excitement of science and 'widening participation' so that the general public can reduce their risk of developing cancer. Before going to Bristol University, he studied as an undergraduate at Manchester University (UK) and then Oxford University (UK) where he obtained his D.Phil. He then held postdoctoral fellowships in Cancer Studies at Birmingham University Medical School (UK) and then Imperial Cancer Research Fund, London (UK) before going to Bristol, where he obtained his chair in 1993. He has a long standing interest in cancer prevention and, in particular, the mechanisms by which NSAIDs, such as aspirin, and dietary factors, such as fiber, exert their chemopreventive properties and their potential exploitation for novel preventive and therapeutic strategies. His current research investigates the role of the prostaglandin $\mathrm{E}_{2} / \mathrm{COX}-2$ signaling pathways in colorectal tumorigenesis and chemoprevention, identification of novel biomarkers for the early detection of bowel cancer, and cancer stem cells. His recent work also focuses on understanding the mechanisms by which cancer cells adapt to key tumor microenvironmental stresses, such as hypoxia and energy deprivation, to target the tumor microenvironment as a novel chemopreventive and therapeutic approach for bowel cancer.

Q What originally led to your interest in bowel cancer?

I studied genetics as an undergraduate and graduate student and, after getting excited about cancer research and tumor-cell biology through working on tumor viruses in my first postdoctoral position with Phil Gallimore in cancer studies at Birmingham University (UK), went on to a second postdoctoral position in the laboratory of
Walter Bodmer (Imperial Cancer Research Fund, London, UK) to work on bowel cancer. This allowed me to combine my interests in tumor cell biology and genetics as I worked on familial adenomatous polyposis, which I really enjoyed. After my second postdoctoral position I was able to take some of the bowel cancer research to Bristol University (UK) and have been doing bowel cancer research ever since.

*School of Cellular \& Molecular Medicine, Medical Sciences Building, University of Bristol, University Walk, Bristol, BS8 1TD, UK; c.paraskeva@bristol.ac.uk

News \& Views

News

Journal Watch

Interviews 
Q Which researchers have influenced or inspired your research?

Denis Burkitt for his observational insights and detective work leading to the naming of Burkitt's Lymphoma, and his linking lifestyle and lack of dietary fiber to bowel cancer. In addition, Bert Vogelstein for his inspirational work on the molecular biology of colorectal cancer, which has formed the paradigm for a great deal of modern cancer research and been highly relevant for both sporadic and hereditary bowel cancers and, importantly, cancer in general.

Q What would you consider to be your biggest achievement in the field?

Studying the mechanism(s) of action and molecular targets of chemoprevention agents. In Bristol we provided the first experimental evidence that chemopreventive agents, such as dietary fiber, and NSAIDs, such as aspirin, may act, at least in part, through inducing apoptosis (programmed cell death). These studies also had relevance regarding the potential of using NSAIDs as adjuvants for treating bowel cancer, as well as in preventing it.

Q You are Director of the Cancer Research UK Colorectal Tumor Biology Research Group at the University of Bristol; could you briefly describe the group's research interests?

Together with Ann Williams, who jointly runs the Colorectal Tumour Biology Group with me, our current work investigates the cross-talk between the Wnt/ $\beta$-catenin and COX-2/prostaglandin E2 (PGE 2$)$ signaling pathways, the fluctuating tumor microenvironment and colon-cancer stem cell biology. One objective is to be able to target adenoma and cancer stem cells with NSAIDs, such as aspirin, and dietary factors, such as dietary fiber. It is hoped that this work will lead to new diagnostic and prognostic markers, as well as identify new targets for prevention and treatment of colorectal cancer. Our work has also led us to investigate the link between inflammation and colorectal cancer, as we have found that modifiers of signaling pathways, such as $\mathrm{NF}-\kappa \mathrm{B}$, play an important part in promoting colorectal tumor cell survival. We are currently involved in translational studies with our clinical colleagues in Bristol, investigating the potential of prostaglandins as biomarkers for colorectal cancer and the use of NSAIDs as adjuvants in pre- and post-operative therapy for cancer treatment, as well as in prevention.

Q You have recently published research on the role of $\beta$-catenin in regulating 15-prostaglandin dehydrogenase. Briefly, what were the main findings and what is the clinical significance of the results?

COX-2 is overexpressed in colorectal cancer and its product, $\mathrm{PGE}_{2}$, plays a pivotal role in promoting colorectal tumorigenesis. Recent studies have revealed that $\mathrm{PGE}_{2}$ levels, as well as being regulated by COX enzymes, are also regulated by the prostaglandin catabolizing enzyme 15-prostaglandin dehydrogenase (15-PGDH), which inactivates $\mathrm{PGE}_{2}$. In the Gut paper, we show that $\beta$-catenin, which is deregulated at a very early stage in the majority of colorectal tumors, represses 15-PGDH expression in colorectal tumor cells [1]. Thus, we report a novel mechanism by which $\beta$-catenin can increase $\mathrm{PGE}_{2}$ levels through downregulating a key tumor suppressor gene, 15-PGDH, and hypothesize that this can occur through COX1 -derived prostaglandins. As Wnt/ $\beta$-catenin signaling initiates most colorectal cancers, our findings suggest that 15-PGDH downregulation will be a widespread and very early change in colorectal neoplasia and, therefore, a highly attractive target for colorectal cancer chemoprevention. Furthermore, targeting both COX isoforms may be required for effective early-stage colorectal chemoprevention. This may explain why aspirin, a nonselective NSAID, is efficient in cancer chemoprevention.

Q What do you see as the hot topics for bowel cancer research over the coming years? The recent identification of normal and cancer functional intestinal stem cell markers is exciting. Although complicated, because of tumor cell 'plasticity', it is hoped these scientific breakthroughs can be exploited for novel chemopreventive and therapeutic strategies and even tissue engineering. Understanding the importance of tumor cells adapting to the tumor microenvironment and its relevance to tumor plasticity/resistance to therapy is clearly important, and may be key to understanding why some metastatic tumors appear to be dormant, only to resurface many years after the patient's treatment has stopped. Although more complex than, for example, the relationship between smoking and lung cancer, 
it is important to better understand the relationship between diet and cancer, since up to $80 \%$ of bowel cancers are preventable by changes in diet. As well as research on diet being important, the scientific/clinical community can, hopefully, help through a similar mechanism of education to promote a change in public attitudes/policy to diet, to help prevent bowel cancer as we have with lung cancer by reducing smoking in public.

Financial \& competing interests disclosure $C$ Paraskeva has no relevant affiliations or financial involvement with any organization or entity with a financial interest in or financial conflict with the subject matter or materials discussed in the manuscript. This includes employment, consultancies, honoraria, stock ownership or options, expert testimony, grants or patents received or pending, or royalties.

No writing assistance was utilized in the production of this manuscript.

\section{Reference}

1 Smartt HJ, Greenhough A, Ordóñez-Morán P et al. $\beta$-catenin represses expression of the tumour suppressor 15-prostaglandin dehydrogenase in the normal intestinal epithelium and colorectal tumour cells. Gut 61(9), 1306-1314 (2012). 